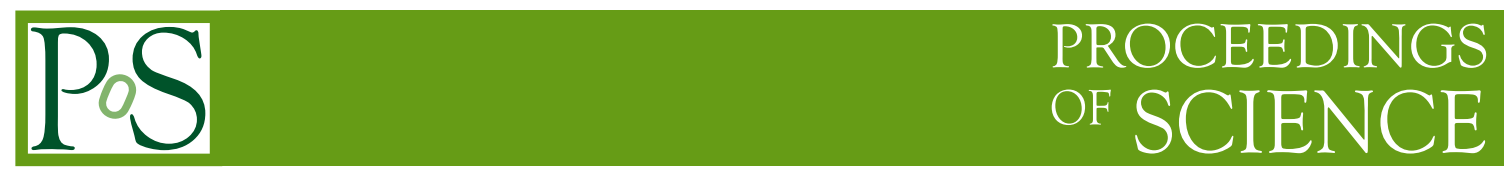

\title{
Observation of primary cosmic rays with the new Tibet hybrid experiment (YAC-II + Tibet-III + MD)
}

M. Amenomori ${ }^{1}$, X. J. Bi ${ }^{2}$, D. Chen ${ }^{3}$, T. L. Chen ${ }^{4}$, W. Y. Chen ${ }^{2}$, S. W. Cui ${ }^{5}$, Danzengluobu $^{4}$, L. K. Ding ${ }^{2}$, C. F. Feng ${ }^{6}$, Zhaoyang Feng ${ }^{2}$, Z. Y. Feng ${ }^{7}$, Q. B. Gou ${ }^{2}$, Y. Q. Guo ${ }^{2}$, H. H. He ${ }^{2}$, Z. T. He ${ }^{5}$, K. Hibino ${ }^{8}$, N. Hotta ${ }^{9}$, Haibing $\mathrm{Hu}^{4}$, H. B. $\mathrm{Hu}^{2}$, J. Huang ${ }^{* 2}$, H. Y. Jia ${ }^{7}$, L. Jiang ${ }^{2}$, F. Kajino ${ }^{10}$, K. Kasahara ${ }^{11}$, Y. Katayose ${ }^{12}$, C. Kato ${ }^{13}$, K. Kawata ${ }^{14}$, M. Kozai ${ }^{13}$, Labaciren ${ }^{4}$, G. M. Le ${ }^{15}$, A. F. Li ${ }^{16,6,2}$, H. J. Li ${ }^{4}$, W. J. Li ${ }^{2,7}$, C. Liu $^{2}$, J. S. Liu ${ }^{2}$, M. Y. Liu ${ }^{4}$, H. Lu ${ }^{2}$, X. R. Meng ${ }^{4}$, T. Miyazaki ${ }^{13}$, K. Mizutani ${ }^{11,17}$, K. Munakata ${ }^{13}$, T. Nakajima ${ }^{13}$, Y. Nakamura ${ }^{13}$, H. Nanjo ${ }^{1}$, M. Nishizawa ${ }^{18}$, T. Niwa ${ }^{13}$, M. Ohnishi ${ }^{14}$, I. Ohta ${ }^{19}$, S. Ozawa ${ }^{11}$, X. L. Qian ${ }^{6,2}$, X. B. Qu ${ }^{26}$, T. Saito ${ }^{20}$, T. Y. Saito ${ }^{21}$, M. Sakata ${ }^{10}$, T. K. Sako ${ }^{14}$, J. Shao ${ }^{2,6}$, M. Shibata ${ }^{12}$, A. Shiomi ${ }^{22}$, T. Shirai ${ }^{8}$, H. Sugimoto ${ }^{23}$, M. Takita ${ }^{14}$, Y. H. Tan ${ }^{2}$, N. Tateyama ${ }^{8}$, S. Torii ${ }^{11}$, H. Tsuchiya ${ }^{24}$, S. Udo ${ }^{8}$, H. Wang ${ }^{2}$, H. R. Wu ${ }^{2}$, L. Xue ${ }^{6}$, Y. Yamamoto ${ }^{10}$, K. Yamauchi ${ }^{12}$, Z. Yang ${ }^{2}$, S. Yasue ${ }^{25}$, A. F. Yuan ${ }^{4}$, T. Yuda ${ }^{14}$, L. M. Zhai ${ }^{2,3}$, H. M. Zhang ${ }^{2}$, J. L. Zhang ${ }^{2}$, X. Y. Zhang ${ }^{6}$, Y. Zhang ${ }^{2}$, Yi Zhang ${ }^{2}$, Ying Zhang ${ }^{2}$ Zhaxisangzhu $^{4}$, X. X. Zhou ${ }^{7}$ 


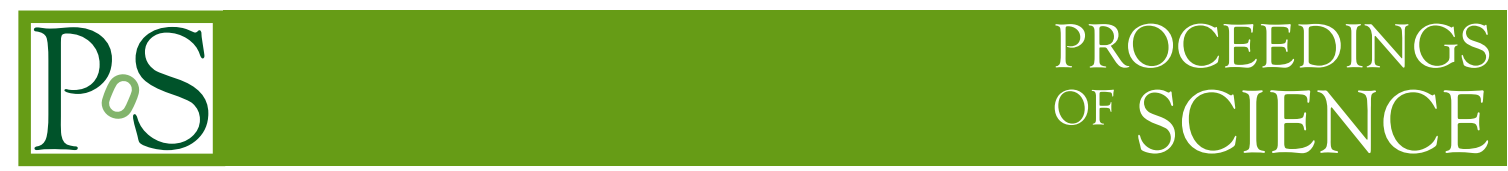

(The Tibet AS $\gamma$ Collaboration)

${ }^{1}$ Department of Physics, Hirosaki University, Hirosaki 036-8561, Japan

${ }^{2}$ Key Laboratory of Particle Astrophysics, Institute of High Energy Physics, Chinese Academy of Sciences, Beijing 100049, China

${ }^{3}$ National Astronomical Observatories, Chinese Academy of Sciences, Beijing 100012, China

${ }^{4}$ Department of Mathematics and Physics, Tibet University, Lhasa 850000, China

${ }^{5}$ Department of Physics, Hebei Normal University, Shijiazhuang 050016, China

${ }^{6}$ Department of Physics, Shandong University, Jinan 250100, China

${ }^{7}$ Institute of Modern Physics, SouthWest Jiaotong University, Chengdu 610031, China

${ }^{8}$ Faculty of Engineering, Kanagawa University, Yokohama 221-8686, Japan

${ }^{9}$ Faculty of Education, Utsunomiya University, Utsunomiya 321-8505, Japan

${ }^{10}$ Department of Physics, Konan University, Kobe 658-8501, Japan

${ }^{11}$ Research Institute for Science and Engineering, Waseda University, Tokyo 169-8555, Japan

${ }^{12}$ Faculty of Engineering, Yokohama National University, Yokohama 240-8501, Japan

${ }^{13}$ Department of Physics, Shinshu University, Matsumoto 390-8621, Japan

${ }^{14}$ Institute for Cosmic Ray Research, University of Tokyo, Kashiwa 277-8582, Japan

${ }^{15}$ National Center for Space Weather, China Meteorological Administration, Beijing 100081, China

${ }^{16}$ School of Information Science and Engineering, Shandong Agriculture University, Taian 271018, China

${ }^{17}$ Saitama University, Saitama 338-8570, Japan

${ }^{18}$ National Institute of Informatics, Tokyo 101-8430, Japan

${ }^{19}$ Sakushin Gakuin University, Utsunomiya 321-3295, Japan

${ }^{20}$ Tokyo Metropolitan College of Industrial Technology, Tokyo 116-8523, Japan

${ }^{21}$ Max-Planck-Institut für Physik, München D-80805, Deutschland

${ }^{22}$ College of Industrial Technology, Nihon University, Narashino 275-8576, Japan

${ }^{23}$ Shonan Institute of Technology, Fujisawa 251-8511, Japan

${ }^{24}$ Japan Atomic Energy Agency, Tokai-mura 319-1195, Japan

${ }^{25}$ School of General Education, Shinshu University, Matsumoto 390-8621, Japan

${ }^{26}$ College of Science, China University of Petroleum, Qingdao, 266555, China

E-mail: huangiingdihep.ac.cn

We have started a new hybrid air shower experiment in Yangbajing (4300 $\mathrm{m}$ a.s.1.) in Tibet since February 2014. This new hybrid experiment consists of the YAC-II comprised of 124 core detectors placed in the form of a square grid of $1.9 \mathrm{~m}$ spacing covering about $500 \mathrm{~m}^{2}$, the Tibet-III AS array with the total area of about $50,000 \mathrm{~m}^{2}$ and the underground MD array consists of 80 cells, with the total area of $\sim 4,500 \mathrm{~m}^{2}$. This hybrid-array system is used to observe air showers of high energy celestial gamma-ray origin and those of nuclear-component origin. Prototype experiment YAC-I was successfully carried out in 2009-2010 together with Tibet-III air-shower array. In this paper, performance of YAC-II and the preliminary results of YAC-I are presented.

The 34th International Cosmic Ray Conference,

30 July- 6 August, 2015

The Hague, The Netherlands

\footnotetext{
*Speaker.
} 


\section{Introduction}

A steep bending of the power spectrum of primary cosmic rays (CRs) at about $4 \times 10^{15} \mathrm{eV}$, called a sharp knee, has been clearly observed with the Tibet air shower experiment [W] and also supported by other experiments. This should be surely related to the origin and propagation of CRs in the Galaxy, but discussions are still under debates. The difficulty of settling discussions is due to a lack of the data on the energy spectra of individual CR components whose energies are too high to catch directly with detectors onboard balloons and satellites because of extremely scarce flux. The early works doing the research of the chemical composition of the knee region were the Tibet emulsion chamber (Tibet-EC) experiment [[] ] and the KASCADE experiment [3]]. The results of the Tibet-EC suggest that the main component responsible for making the knee structure is composed of nuclei heavier than helium, however, the KASCADE claims that the knee is due to the steepening of the spectra of light elements with an exponential type cutoff as shown in Fig.D. It is also noted that the experimental data points are still poor in the energy range between the direct measurements and the indirect measurements waiting us to study. In order to explicitly observe the break point of the spectral index for individual chemical component, we have recently made the upgrade of the Tibet-EC experiment and started a new Tibet hybrid experiment (YAC-II+TibetIII+MD). This new hybrid experiment aims to observe the energy spectrum of Proton, Helium and Iron whose energy range will overlap with direct observations at lower energies such as CREAM, ATIC and TRACER, and Tibet-EC experiment at higher energies. On the other hand, we add underground muon detector to new Tibet hybrid experiment for measuring the muon component contained in the air showers at the high attitude where dependence on hadronic interaction models is expected to be much less than the cases of the sea-level observations. In this paper, we performed a full Monte Carlo simulation to check the sensitivity of YAC to the interaction models and primary CR models both of which contain uncertainties.

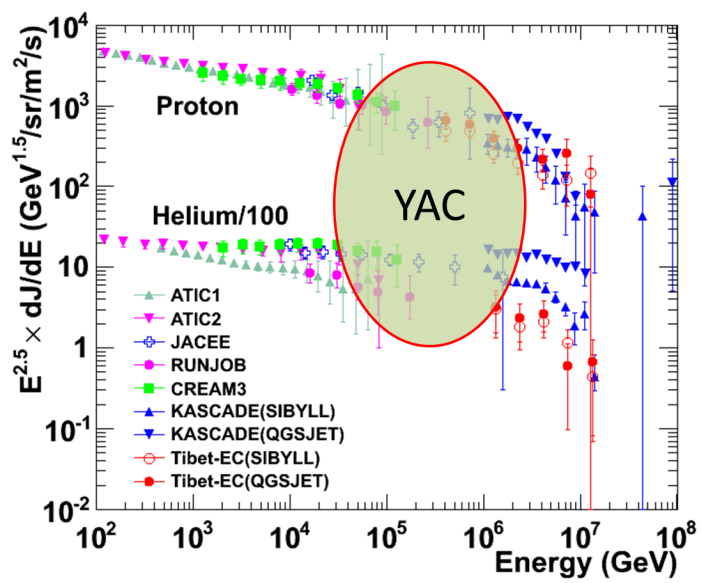

Figure 1: Energy spectra of primary cosmic-ray protons and helium nuclei obtained by Tibet-ECs and they are compared with other experiments.

\section{The experimental setup}

The new Tibet hybrid experiment currently consists of three types of detector array, including 


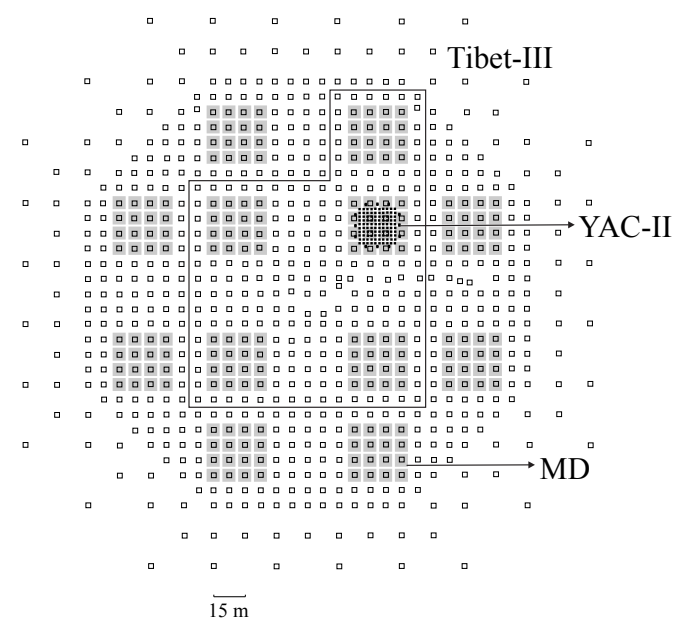

Figure 2: Schematic view of (Tibet-III+YAC-II+MD) array.

the Tibet AS array (Tibet-III), the Yangbajing AS core-detector array (YAC-II) and an underground water-Cherenkov muon-detector array (MD), as shown in Fig.Z. The Tibet-III consists of 789 detector units, with a covering area $\sim 50,000 \mathrm{~m}^{2}$; the YAC-II consists of 124 detector units, with a covering area of $\sim 500 \mathrm{~m}^{2}$ and the five MD pools consists of 80 cells, with an area of $\sim 4,500 \mathrm{~m}^{2}$ in total. This new hybrid experiment (Tibet-III+YAC-II+MD) has been constructed and operated at Yangbajing (4300 $\mathrm{m}$ above sea level; $606 \mathrm{~g} / \mathrm{cm}^{2}$ ) in Tibet, China, and data taking started from February 2014. The detail description about this hybrid experiment could be seen in paper [ [4].

The YAC-II array is constructed near the center of the Tibet-III as shown in Fig.Z. For an air shower event, the Tibet-III provides the arrival direction $(\theta)$ and the air shower size $\left(N_{e}\right)$ which are interrelated to primary energy, the YAC-II measures the high energy electromagnetic particles in the core region so as to obtain the characteristic parameters of air-shower cores, at the same time, the underground MDs record the high-energy muons above $1 \mathrm{GeV}$. When a YAC event is triggered, its accompanying air shower is simultaneously recorded. The matching between YAC, AS and MD event is made by their arrival time stamps.

\section{Monte Carlo Simulations}

We have carried out a full Monte Carlo (MC) simulation on the development of air showers in the atmosphere using the simulation code Corsika(version 7.3500) [G] including the newest interaction models EPOS-LHC(v.3400), QGSJETII-04 and SIBYLL2.1. For the primary cosmic rays, we examined three primary composition models (shown in Fig.[B]), namely, "He-poor", "Herich" and "Gaisser-fit" models, in order to evaluate the systematic errors attributable to primary composition models [四, []. The proton spectra of the former two models are fitted to the direct measurements at the low energy and consistent with the spectrum obtained from the Tibet-EC experiment at the high energy. The He spectrum of He-poor model coincides with the results from RUNJOB, but the He spectrum of He-rich model coincides with the results from JACEE, ATIC2 and CREAM. The Gaisser-fit model fits to a higher He model (almost same as our Herich model) at the low energy range and to the KASCADE-QGSJET data at high energy range 


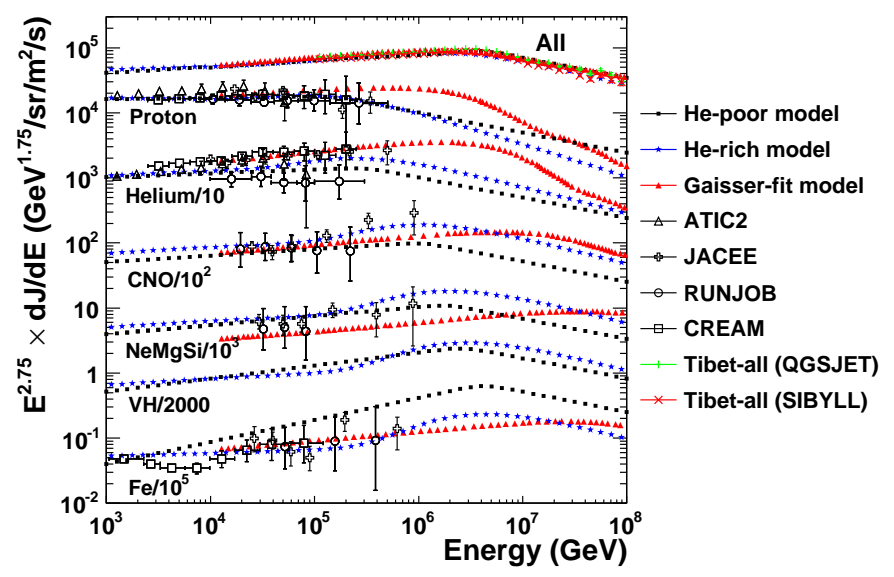

Figure 3: Primary cosmic-ray composition for He-poor, He-rich and Gaisser-fit models compared with those of direct measurements [[]] and the sum of all components compared with the results obtained by the Tibet-III experiment [四 .

in which light components ( $\mathrm{P}$ and $\mathrm{He}$ ) dominate in the chemical composition [B]. In this three composition models mentioned above, each component is summed up so as to match with the all-particle spectrum with a sharp knee, which was obtained with the Tibet-III AS array [四]. The energy spectra of individual components (or mass groups) for three primary models are shown in Fig. [3. It is seen that all the individual components of the three models in the low energy range (less than $100 \mathrm{TeV}$ ) are in good agreement with direct measurements while differ significantly at higher energy. The all-particle spectra of three models, however, coincide with each other and reproduce the sharp knee [四] structure as well.

The detector responses to shower particles falling on the detectors of (YAC-II+Tibet-III+MD) array are calculated using the Geant $4[\square]$, where the detector performance, trigger efficiency and effective area are adequately taken into account based on the experimental conditions. After reconstruction of air shower, we can obtain some characteristic parameters observed with the (YACII+Tibet-III+MD) array, and we find these parameters are very sensitive to different primary CR components.

\section{Results and Discussion}

\subsection{Expected primary proton, helium and iron spectra}

In this work, we use a feed-forward artificial neural network (ANN) method [ [ $]$ ] to separate P-like events, He-like events and Iron-like events by using the above characteristic parameters observed with the (YAC-II+Tibet-III+MD) array. Fig.⿴囗十 shows that this hybrid experiment has a capability of measuring the primary energy spectrum for each of 4 mass groups of $\mathrm{P}, \mathrm{He}$, Iron and $4<\mathrm{A}<56$ at $50 \mathrm{TeV}-10 \mathrm{PeV}$ range covering the knee with an energy resolution better than $12 \%$ at $10^{15} \mathrm{eV}$. This enables us to find the break energies of the power spectra of protons and helium nuclei since these are the main components in the knee region. This is indispensable to deepen our understanding on the origin and acceleration mechanism of primary CRs up to the knee energy. 

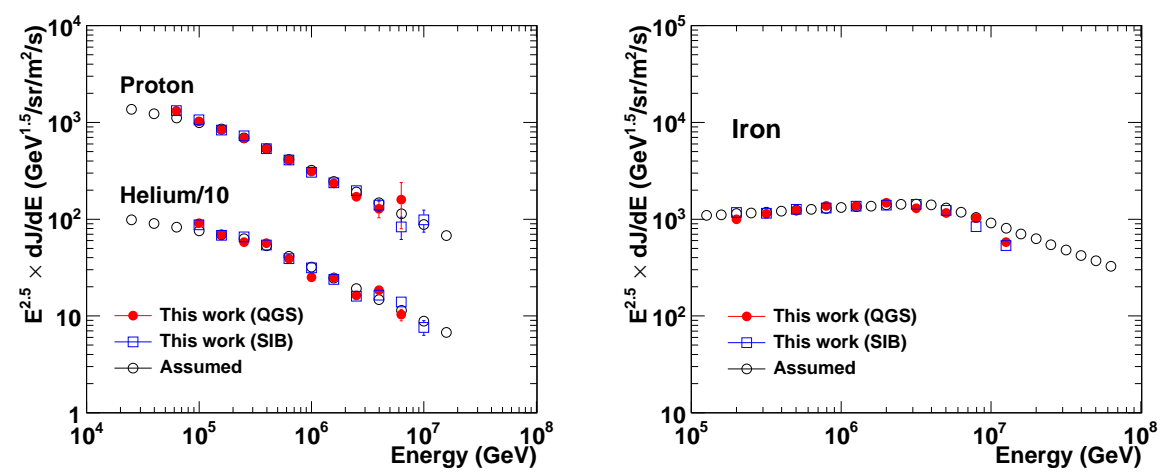

Figure 4: The expected energy spectra of primary Proton, Helium (left Fig.) and Iron (right Fig.) by ANN method compared with the inputs to the simulation based on "He-poor" model.

\subsection{Test of hadronic interaction models by (YAC-I+Tibet-III) hybrid experiment}

The interpretation of the air showers is known to inevitably depend on the Monte Carlo simulations which are based on some hadronic interaction models and cosmic-ray composition models. At present, the simulation code CORSIKA that is comprehensively used in the ground-level cosmic-ray studies includes many interaction models. In this paper, we report our approach and the preliminary results to check the hadronic interaction models EPOS-LHC, QGSJETII-04 and SIBYLL2.1 at an energy region of $100 \mathrm{TeV}-1 \mathrm{PeV}$ using the data obtained by the prototype YACI (Yangbajing Air shower Core detector as the first stage). Data taking was made from May 2009 to February 2010, together with the Tibet-III array.

This prototype 16 YAC-I detectors were constructed near the center of the Tibet-III AS array with dense spacing. The response linearity of each YAC-I detector was calibrated by cosmicray single muons and by the accelerator beam (BEPC-LINAC) [9]. YAC-I is triggered when any one of 16 detectors records a local shower with the size of at least 20 MIPs. The event rate is about $30 \mathrm{~Hz}$. The total live time of our data set in present analysis is 169.65 days. Full MC simulation was made using three interaction models of EPOS-LHC, QGSJETII-04 and SIBYLL2.1 with primary energy above $5 \times 10^{13} \mathrm{eV}$. We examined three primary composition models namely, "He-poor", "He-rich" and "Gaisser-fit" models (shown in Fig.b ). All detector responses is based on the detector simulation code Geant4(version 9.5). The simulated events are passed through the same analysis chains as the experimental data. The statistics of high energy EAS core events are shown in Tablell.

YAC-I shows the ability and sensitivity in checking the hadronic interaction models in 100 $\mathrm{TeV}$ - $1 \mathrm{PeV}$ region. Following quantities of YAC-I are used to characterize an high energy AS core event: high-energy electromagnetic components (electrons and photons) in the AS core induces cascade showers in lead absorber of the YAC detector (thickness of $3.5 \mathrm{~cm}$ ). The number of shower electrons under the lead layer is measured by scintillator equipped with PMTs and called 'burst size' $\left(N_{b}\right)$. The conversion from PMT signal to particle number is calibrated by single muon peak. When the burst size of a detector unit is higher than 100 , this unit is defined as a 'fired' one. We also call the total burst size of all fired detector units as $\sum N_{b}$, the maximum burst size among fired detectors as $N_{b}^{t o p}$, and the mean lateral distance as $\langle R\rangle$, and the mean $N_{b}$ weighted 
Table 1: Statistics of EAS core events in MC simulation and Experiment.

\begin{tabular}{ll}
\hline \hline Models & Core events \\
\hline SIBYLL2.1+He-rich & 64,331 \\
SIBYLL2.1+He-poor & 47,580 \\
QGSJETII-04+He-poor & 31,928 \\
EPOS-LHC+He-poor & 42,137 \\
EPOS-LHC+Gaisser-fit & 139,098 \\
EPOS-LHC+He-rich & 246,938 \\
\hline Expt.data & 5035 \\
\hline
\end{tabular}
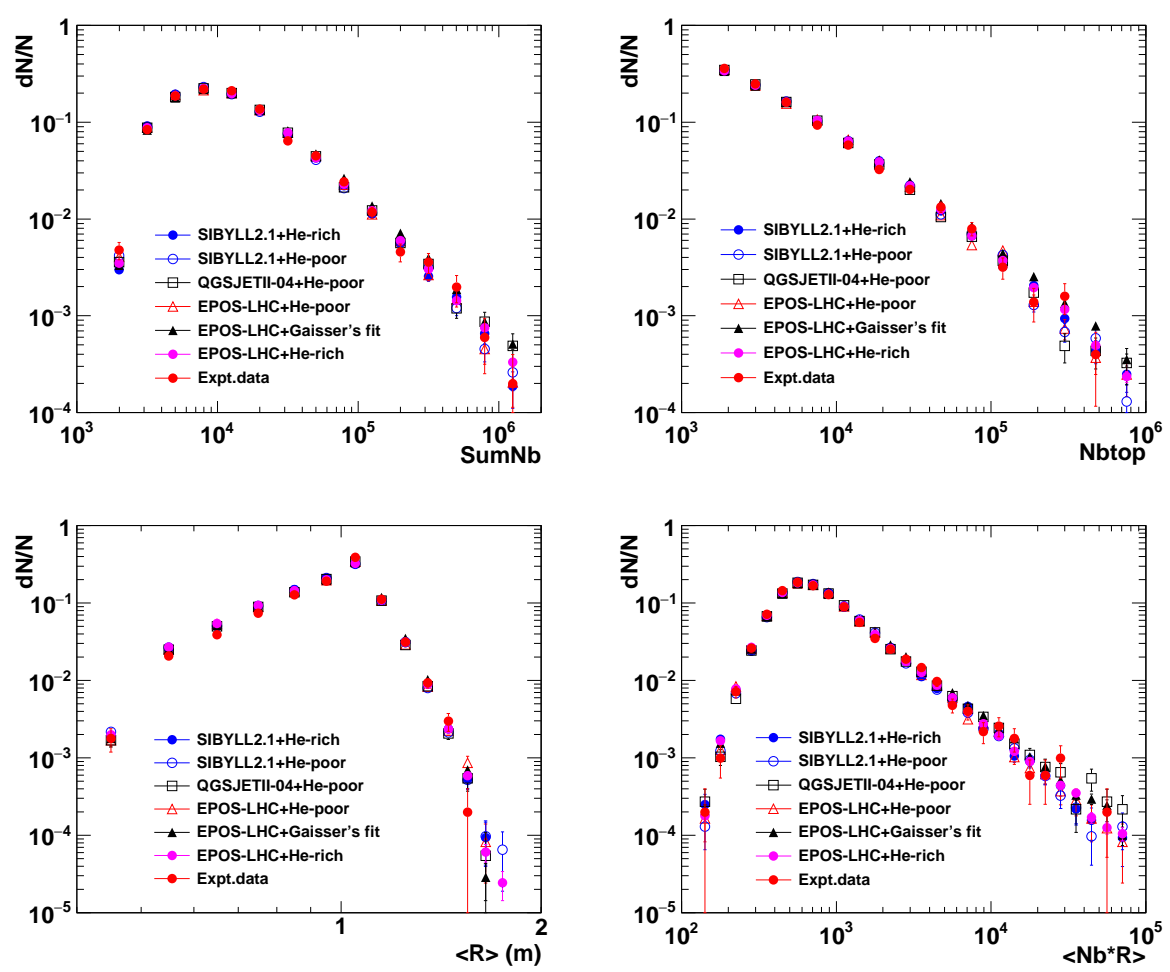

Figure 5: The comparison of some observable parameters $\left(\sum N_{b}, N_{b}{ }^{t o p},\langle R\rangle,\left\langle N_{b} R\right\rangle\right)$ between experimental data and MC simulation which are calculated for various combination of interaction and composition models.

lateral distance as $\left\langle N_{b} R\right\rangle$. The comparison of some observable parameters between YAC-I experimental data and $\mathrm{MC}$ simulation data which are calculated for various combination of interaction and primary composition models are seen from Fig.5 and Fig.6:

Fig. 5 shows that the shape of each parameter distribution is almost same since these AS-core events are mainly a superposition of many EM cascade processes originated by decayed gamma rays from neutral pions. YAC-I experimental data are consistent with those MC simulation results, which means that there exist no detection bias within our experimental systematic uncertainty of a level about $10 \%$. Please note that the shape shows a (cascade) scaling behavior, while its intensity 

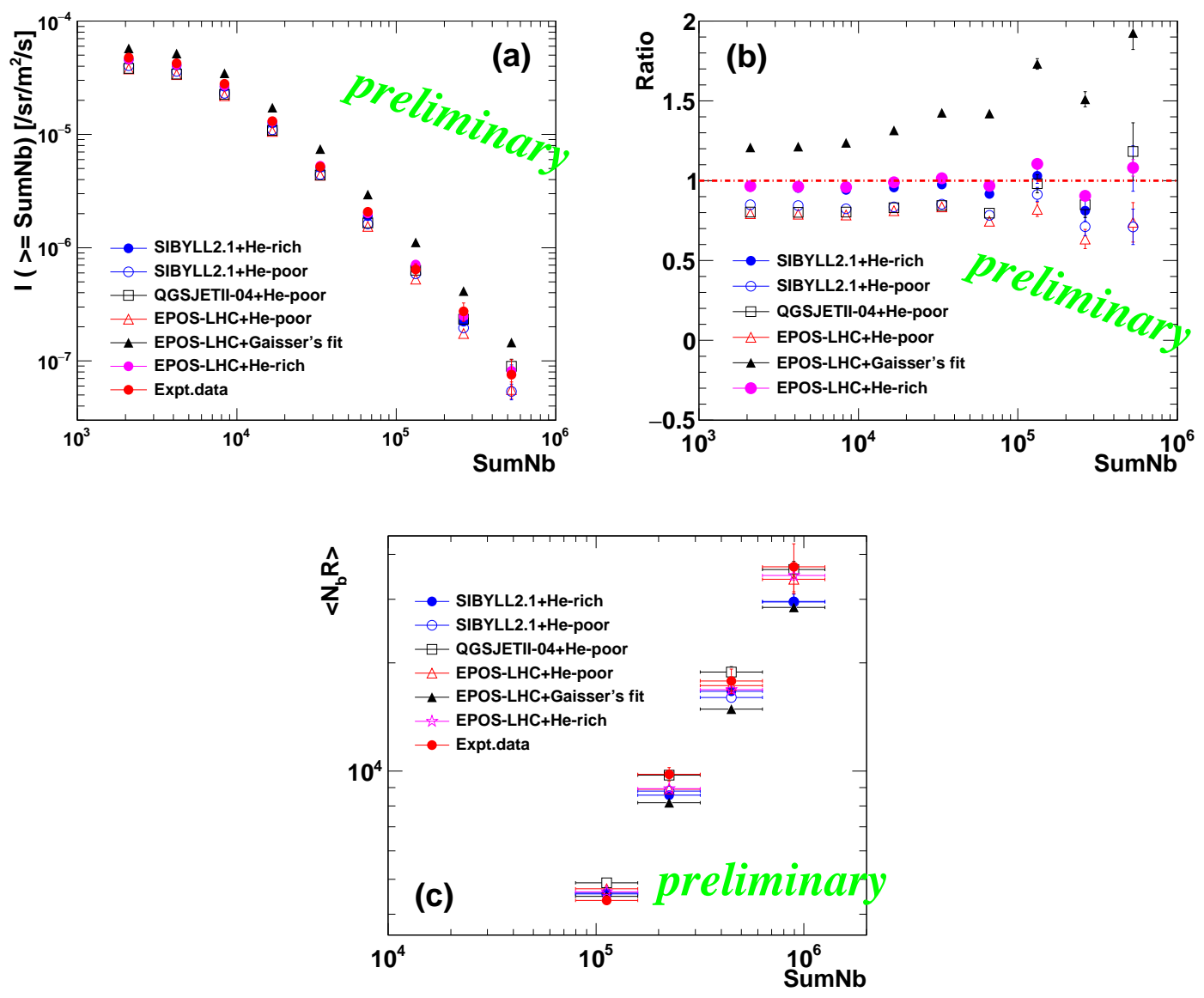

Figure 6: (a) - Integral $\sum N_{b}$ (SumNb) spectra obtained from six MC models and Experimental data, (b) The intensity ratios of $\sum N_{b}$ to that obtained by the Experimental data, the red dash line ( Ratio = 1 ) denoted the Experimental data, (c) - The mean energy-flow lateral spreads $\left\langle N_{b} R\right\rangle$ in the respective energy interval for six MC models and Experimental data.

depends on the primary composition model.

As seen in Fig.6 (a)-(b), there exist some discrepancies in the absolute intensities: 1)first, note that the three interaction models give almost same intensity when the primary is fixed to the "He-poor" model. This results suggest that the difference among three interaction models are very small, say at most $10 \%$ since almost all high energy cores are produced by the same primary-proton spectrum (He contribution is estimated to be very small [团]). 2) experimental data are very close to SIBYLL2.1, EPOS-LHC and QGSJETII-04, if we assumed "He-rich" primary composition. Hence, YAC-experimental data is consistent with primary composition with the "Herich" model, while we can say anything about heavier nuclei than He. 3) the "Gaisser's fit" primary model however always gives a higher flux by a factor $1.4-2.0$ than the experimental result and other primary models. It teaches us that there are too many primary light components $(\mathrm{P}, \mathrm{He})$ in "Gaisser's fit" model than our experimental data.

Mean lateral spreads of AS-cores events are compared with the MC simulation results as shown in Fig.6(c). It is known that although these are not strong, it is dependent on the transverse 
momentum of secondary particles produced in hadronic interactions as well as interaction height as seen in Fig.6(c). Some discrepancies in the mean lateral spreads are seen: YAC-experimental data are very close to "EPOS-LHC+He-rich" and "QGSJETII-04+He-poor" model. However, the "Gaisser's fit" primary composition model always gives a lower distribution than YAC-I experimental results. This conclusion is same as the above Fig.6(a)-(b), that is, there are too many primary light components $(\mathrm{P}, \mathrm{He})$ in "Gaisser's fit" than our experimental data. The above mentioned results well show that YAC is very sensitive to observe primary light components and well reflet the structure of their spectra as noticed in primary composition spectrum as noticed in Fig.6(a),(b) and (c).

\section{Summary}

YAC-I shows the ability and sensitivity in checking the hadronic interaction models. Based on the "He-poor" primary model, we estimate that the difference of EPOS-LHC, QGSJETII-04, SIBYLL2.1 is within $10 \%$ in our concerned energy region. High core events are very sensitive to the light components in CRs and the core parameters are very useful to separate the light components from all the observed events using a ANN technique. Simultaneous observation of core events with YAC and MD array may allow us to obtain the spectrum of each of primary components separately. Actually, the full MC simulation shows that the (YAC-II+Tibet-III +MD) array is powerful enough to obtain the energy spectra of proton, helium, medium nuclei and iron spectra of primary CRs in the range of $50 \mathrm{TeV}-10 \mathrm{PeV}$ with sufficient accuracy. It is estimated that the energy resolution of primary particles of $1 \mathrm{PeV}$ is better than $12 \%$.

\section{Acknowledgments}

The collaborative experiment of the Tibet Air shower Arrays has been performed under the auspices of the Ministry of Science and Technology of China and the Ministry of Foreign Affairs of Japan. This work is supported by the Grants from the National Natural Science Foundation of China (11078002 and 11275212) and the Chinese Academy of Sciences (H9291450S3, 2013T2J0006) and the Key Laboratory of Particle Astrophysics, Institute of High Energy Physics, CAS. The Knowledge Innovation Fund (H95451D0U2 and H8515530U1) of IHEP, China also provide support to this study.

\section{References}

[1] M. Amenomori et al., ApJ 678 (2008) 1165

[2] M. Amenomori et al., Phys. Lett. B 632 (2006) 58

[3] T. Antoni et al., Astropart. Phys. 24 (2005) 1

[4] J. Huang et al., Astropart. Phys. 66 (2015) 18

[5] L. M. Zhai et al., J. Phys. G: Nucl. Part. Phys. 42 (2015) 045201

[6] Heck D. et al., 1998 Forschungszentrum Karlsruhe Report FZKA 6019

[7] Agostinelli S. et al., Nucl. Instrum. Meth. Phys. Res. A 506 (2003) 250

[8] Peterson C. et al., 1994 Comp. Phys. Comm. 81185

[9] M.Amenomori, et al., The Proc. 32nd Int. Cosmic Ray Conf. (Beijing) (HE 1.4,ID: 1217), (2011). 